\title{
Paediatric Bone and Joint Infections: causative organisms and antibiotic treatment
}

\section{Singer E., Beuvink Y., Makwana N.}

\section{Sandwell and West Birmingham Hospitals NHS Trust, Birmingham, UK}

\section{Background}

Osteomyelitis (OM) is uncommon in children in Western populations ${ }^{1}$ Resistant and virulent organisms including MRSA and Panton-Valentine Leukocidin-producing (PVL) Staphylococcus aureus, which causes leucocyte destruction and tissue necrosis, make effective treatment challenging ${ }^{2}$, often requiring the use of longer courses of multiple antibiotics, some with toxic side effect profiles. Current data suggest the incidence of PVL-producing Staphylococcus Aureus is low in the UK and more commonly associated with MSSA than in North America $^{3}$. This epidemiological evidence also suggests that children with PVL positive musculoskeletal infections are more likely to require surgical intervention.

Aim: to establish the incidence of causative organisms in OM \& the antimicrobial therapy used, including agent \& duration.

\section{Methods}

31 paediatric patients from Sandwell and West Birmingham Hospitals NHS Trust coded as OM or SA between 2010 and 2016 were identified retrospectively. Data were accessed from casenotes $(n=21)$ and electronically.

Email: eleanor.singer@nhs.net

Rates of OM or SA caused by PVLproducing Staphylococcus aureus were compared to recent literature and patients' treatment reviewed.

\section{Results}

There were 31 patients treated empirically for OM. 23 of the presentations were radiologically confirmed cases of OM. The age range was 2 weeks - 16 years. (Median 8 years). Positive microbiology included blood cultures $(n=8)$ and Intra-operative samples $(n=11)$. The PVL status of intra-operative samples is unknown. MSSA was the most common organism. 5 patients had PVL-producing staph. aureus in blood cultures. (Fig. 1).

All patients with PVL-producing staphylococcus infections required operative management; 2 had multi-focal disease. These patients also had comparatively higher inflammatory markers than the other cases. All patients with PVLproducing MSSA strains received antibiotic combination therapy with at least three iv antibiotics. Flucloxacillin was part of the regime in all cases (Fig. 2). These patients also required longer antibiotic courses (median 12 weeks vs 6 weeks for non-PVL).

\begin{tabular}{|c|c|}
\hline Culture result & $n$ \\
\hline Blood culture & 25 \\
\hline \multicolumn{2}{|l|}{ Staphylococcus aureus } \\
\hline MSSA & 5 \\
\hline MRSA & 1 \\
\hline PVL-producer & 5 (including MRSA) \\
\hline \multicolumn{2}{|l|}{ Streptococci } \\
\hline Strep. pneumoniae & 1 \\
\hline Strep. agalactiae & 1 \\
\hline Sterile & 17 \\
\hline $\begin{array}{l}\text { Intra-operative sample } \\
\text { (Not tested for PVL) }\end{array}$ & 11 \\
\hline MSSA & 10 \\
\hline MRSA & 1 \\
\hline
\end{tabular}
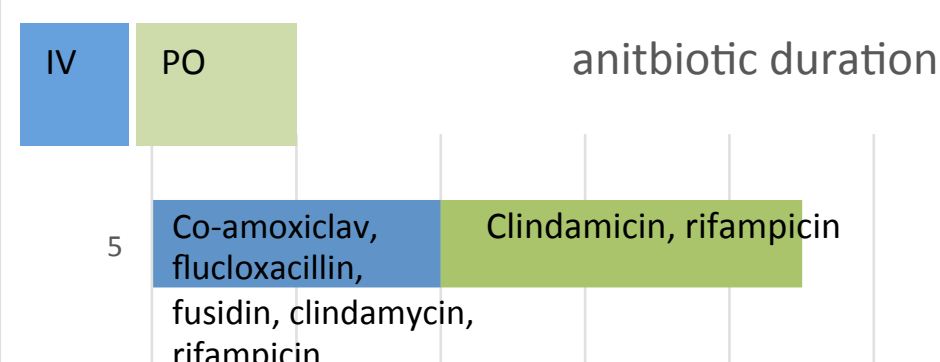

ifampicin

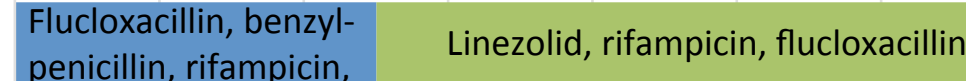
penicillin,
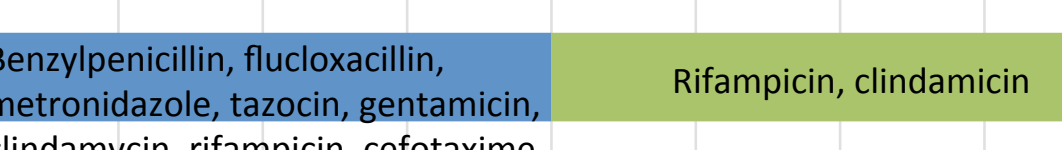

ampicin, clindamici
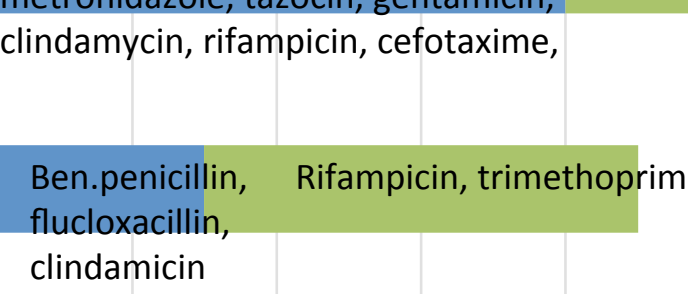

Flucloxacillin, clindamicin Unknown, pt transferred for care

\section{Conclusion}

$83 \%$ of staphylococci in blood cultures were PVL-producers, a higher proportion than suggested by other European data ${ }^{3}$. These patients provide a treatment challenge, often requiring broader-spectrum antibiotic therapy. Therefore, obtaining samples for culture and testing for PVL-production is essential to optimise therapy. Our patients had no long-term disability, though 2 of 5 patients had multi-focal disease. Sample size was small, however, our results correlate with the previously demonstrated low incidence of PVL-positive MRSA in Britain ${ }^{3}$. Our unit does not routinely test for PVL status in samples other than blood cultures. However, given the relatively high prevalence of this toxin producing organism, clinicians should have a high index of suspicion and test accordingly.

\section{References}

1. Faust SN, Clark J, Pallett A, Clarke NM. Managing bone and joint infection in children. Arch Dis Child. 2012 Jun;97(6): 545-53.

2. Yeo A, Ramachandran M. (2014) Acute haematogenous osteomyelitis in children. British Medical Journal. Jan 20;348:g66. doi: 10.1136/bmj.g66. Review.

3. Shallcross LJ, Fragaszy E, Johnson AM, Hayward AC (2013). The role of the Panton-Valentine leucocidin toxin in staphylococcal disease: a systematic review and metaanalysis. Lancet Infectious Diseases. Jan;13(1):43-54.

\section{Acknowledgements}

We wish to thank $\operatorname{Dr}$ T. Masand for contribution to data collection; Dr Ratnaraja and the team at the SWBH Microbiology department for advice as well as the SWBH pharmacy and clinical effectiveness departments for their support in this work. 